$19^{\text {th }}$ European Symposium on Computer Aided Process Engineering - ESCAPE19

J. Jeżowski and J. Thullie (Editors)

(C) 2009 Elsevier B.V./Ltd. All rights reserved.

\title{
Design and Control of New Intensified Distillation Systems for Quaternary Separations using Genetic Algorithms
}

\author{
José Antonio Vázquez - Castilloa , Josué Addiel Venegas - Sáncheza , Juan \\ Gabriel Segovia - Hernándeza , Salvador Hernández $z^{a}$, Héctor Hernández \\ Claudia Gutiérrez - Antonio ${ }^{\mathrm{b}}$ and Abel Briones - Ramírez ${ }^{\mathrm{c}, \mathrm{d}}$ \\ ${ }^{a}$ Universidad de Guanajuato, Departamento de Ingeniería Química, Noria Alta $s / n$, \\ Guanajuato, Gto., México 36050,gsegovia@quijote.ugto.mx \\ ${ }^{b}$ CIATEQ, A.C., Av. del Retablo 150, Col. Fovissste, Querétaro, Querétaro, México, \\ 76150. \\ ${ }^{c}$ Instituto Tecnológico de Aguascalientes, Departamento de Ingeniería Química, Av. \\ Adolfo López Mateos \#1801 Ote. Fracc. Bonagens, Aguascalientes, Aguascalientes, \\ Méxic, 20256. \\ ${ }^{d}$ Innovación Integral de Sistemas S.A. de C.V., Limas No. 5 Manzana C, \\ Fraccionamiento Don Manuel, Querétaro, Querétaro, México, 76114.
}

\begin{abstract}
The design and optimization of a coupled distillation system is a non-linear and multivariable problem. The complexity of this kind of problem leads to the high difficulty for solving it. This paper addresses the application of genetic algorithms to the optimization of intensified distillation systems for quaternary distillations. For that purpose, we used a multi-objective genetic algorithm with restrictions, written in Matlab ${ }^{\mathrm{TM}}$ coupled to process simulator Aspen Plus ${ }^{\mathrm{TM}}$ for the evaluation of the objective function.
\end{abstract}

Keywords: Genetic algorithms, dividing wall columns, design, control properties.

\section{Introduction}

Process intensification (PI) is an area presently receiving considerable interest in the chemical engineering. Stankiewicz and Moulijn [1] provide a definition of PI, comprising novel equipment, processing techniques, and process development methods that, compared to conventional ones, offer substantial improvements in (bio)chemical manufacturing and processing as well as an extensive description of a PI toolbox, ordered along two dimensions: equipment and processing methods. Distillation handles about $3 \%$ of the total US energy consumption, more than $90 \%$ of all product recovery and purification separations in US and more than $95 \%$ of chemical industries consumption worldwide. Distillation processes are highly energy-consuming systems, and any small improvement in distillation can provide huge energy savings. Thermally coupled distillation sequences (TCDS) are an example of process intensification. Thermal coupling has been used in the design of multicomponent distillation systems to significantly reduce both energy consumption and capital costs when compared with conventional simple column configurations. Thermally coupled distillation sequences are an example of process intensification. Specifically, the thermally coupled dividing wall column (DWC) has been successfully used in many industrial separations for 
ternary mixtures. The DWC offers the possibility of both energy and capital cost savings. Capital cost savings result from a reduction in quantity of equipment (i.e., one shell instead of two in the case of the Petlyuk column). There are also indirect benefits: a DWC requires less plot area, and, therefore, shorter piping and electrical runs. Flare loads are reduced because of the lower heat input and smaller fire-case surface, leading to a smaller flare system. Amminudin et al. [2] reported the industrial acceptance and commercialization of DWC by organizations such as BASF AG, M.W. Kellogg (together with BP, later known as BP Amoco), and Sumitomo Heavy Industries Co. together with Kyowa Yuka. Linde AG constructed the world's largest DWC for Sasol, an estimated $107 \mathrm{~m}$ tall and $5 \mathrm{~m}$ in diameter [3]. In 2004, Adrian et al. [4] reported that BASF operates about 30 DWCs worldwide in their plants. Recently, efforts have focused on finding new thermally coupled configurations based on energy savings as well as operability.

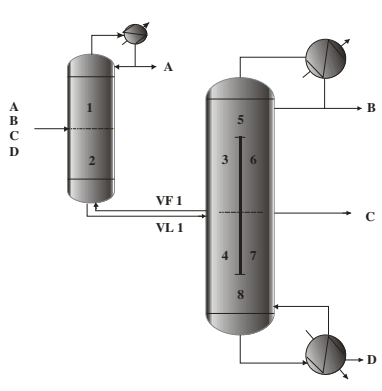

a) DWCS-1

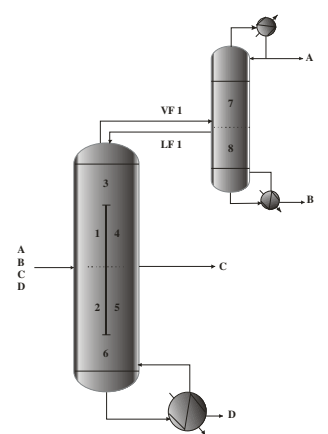

d) DWCS-4

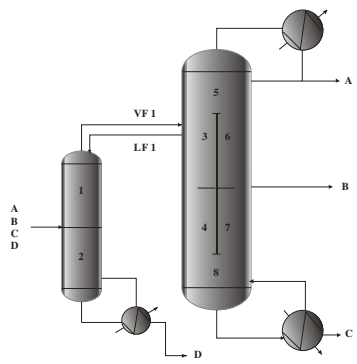

b) DWCS-2.

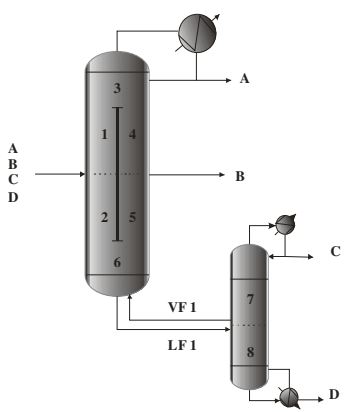

c)

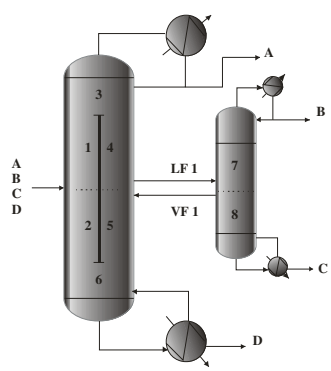

e) DWCS-5

Figure 1. Intensified distillation systems for quaternary separations.

There are few works on extensions toward the design of DWC for mixtures of more than three components. The optimal design of DWC for separation of multicomponent mixtures is a non-linear and multivariable problem, and the objective function used as optimization criterion is generally non-convex with several local optimums. However, the task is complicated and is likely to fail to achieve convergence. In this paper, we have studied the design of five new intensified distillation systems for quaternary 
separations, with dividing walls, (Figure 1) using as a design tool a multi-objective genetic algorithm with restrictions coupled with the process simulator Aspen Plus ${ }^{\mathrm{TM}}$ [2] for the evaluation of the objective function, ensuring that all results obtained are rigorous. Numerical performance of this method has been tested in the design of columns with several mixtures to examine the effect of the relative volatilities of the feed mixtures.

\section{Design Tool: Genetic Algorithm}

Recent years have seen increased development and application of global optimization strategies in many areas of chemical engineering. Global optimization methods can be classified as deterministic or stochastic. The first class offers a guarantee of finding the global optimum of the objective function, provided that the objective function is convex. On the other hand, stochastic optimization methods are robust numerical tools that present a reasonable computational effort in the optimization of multivariable functions; they are applicable to ill-structured or unknown structure problems, and can be used with all thermodynamic models. In the case of stochastic optimization, there are methods known as genetic algorithms (GA), which are a part of the wider field of evolutive algorithms. Optimal design of the new intensified distillation systems implies the determination of 16 variables among continues and integers, such as total number of stages, location of feed stages, location of the exits and entrances of interconnection flows, reflux ratio, product and interconnection flows. The elevated number of variables along with the enthalpy and phase equilibrium calculations for quaternary mixtures makes this problem mixed-integer non-lineal. Optimal design for these sequences means having a structure with as few as possible stages and as small as possible heat duty, but satisfying the purities required. The optimization of the intensified distillation systems implies the minimization as the number of stages in each column, $\mathrm{Nj}$, as the heat duty of the reboilers presented in the sequence, Qi, subject to achieve the purities required in each stream product:

$\operatorname{Min}\left(Q_{i}, N_{j}\right)=f\left(R, N_{k}, N_{l}, F_{k}, N_{F}, N_{S}\right)$

subject to

$\vec{y}_{m} \geq \vec{x}_{m}$

Where $\mathrm{R}$ is the reflux ratio, $\mathrm{N}_{\mathrm{k}}$ is the number of stage of the outlet interconnection flow $\mathrm{k}$ in the column $\mathrm{j}, \mathrm{N}_{\mathrm{l}}$ is the number of stage of the inlet interconnection flow 1 in the column $\mathrm{j}, \mathrm{F}_{\mathrm{k}}$ is the value of the interconnection flow $\mathrm{k}, \mathrm{N}_{\mathrm{F}}$ is the number of stage of the feed stream, $\mathrm{N}_{\mathrm{S}}$ is the number of stage of the side stream, and are the vectors of obtained and required purities for the m components, respectively. The sequences DWCS-1 and DWCS-3 have four objectives to minimize: the number of stages in the three columns and the heat duty of the unique reboiler; while in sequences DWCS-2, DWCS-4 and DWCS-5 there are five objectives: those included in the previous sequences plus the heat duty of an additional reboiler. For all sequences, minimization of these objectives includes the manipulation of 16 variables as continuous as integer, which include reflux ratio, location and values of the interconnection flows, and the feed stage of the sequence. These 4 or 5 objectives have to be minimized simultaneously, since they are in competition. 
In order to solve this problem, we used the multiobjective genetic algorithm with constraints coupled to Aspen Plus ${ }^{\mathrm{TM}}$ proposed by Gutiérrez-Antonio et al [5] to manage five and four objectives along with the 4 constraints of purities, depending on the sequence. The multiobjective genetic algorithm used allows obtaining the rigorous Pareto front of the intensified distillation systems: a set of non dominated, optimal and rigorous designs that satisfied the purities required. The term non dominated means that there is no other design that can improve one objective without worsen another one. From that set of non dominated optimal design, we choose one design for each case due to space limitations of the manuscript. The term rigorous means that all designs presented were obtained considering the complete set of MESH equations along with the phase equilibrium calculations, using the module Radfrac of Aspen Plus ${ }^{\mathrm{TM}}$. For all sequences we employ 2000 individuals and 60 generations, as parameters of the algorithm. These parameters were obtained through a tuning process, in which we were looking to ensure the convergence in a reduced number of generations, and seeking diversity in the Pareto front. The time employed for the optimization of each sequence is between 8 and 10 hours on a Xeon 5410 workstation at $2.33 \mathrm{GHz}$ with $8 \mathrm{~GB}$ of RAM. Figure 2 displays a block diagram for the genetic algorithm.

\section{Case of Study}

The description of the mixtures and the composition in the feed used in this paper is given in Table 1; the feed flowrate was $45.36 \mathrm{kmol} / \mathrm{h}$ as saturated liquid, and the specified purities for the product streams were assumed to be 98.7, 98, 98 and 98.6 mole percent for A, B, C and D respectively. The design pressure for each separation was chosen to ensure the use of cooling water in the condensers. Since the feeds M1 and M2 involve a hydrocarbon mixture, the Chao-Seader correlation was used for the prediction of thermodynamic properties. In the case of M3 and M4, the UNIQUAC model was used for calculations.

Table 1. Mixtures analyzed.

\begin{tabular}{lll}
\hline Mixture & $\begin{array}{l}\text { Component } \\
(\mathrm{A}, \mathrm{B}, \mathrm{C}, \mathrm{D})\end{array}$ & $\begin{array}{l}\text { Feed composition } \\
\text { (mol. fraction) }\end{array}$ \\
\hline M1 & n-pentane, n-hexane, n-heptane, n-octane & $0.15,0.35,0.35,0.15$ \\
\hline M2 & $\begin{array}{l}\text { 2-methyl-butane,n-pentane,2-methyl-hexane,n- } \\
\text { hexane }\end{array}$ & $0.15,0.35,0.35,0.15$ \\
\hline M3 & benzene, toluene, ethylbenzene, o-xylene & $0.15,0.35,0.35,0.15$ \\
\hline M4 & methanol, 1-propanol, 1-pentanol, 1-octanol & $0.15,0.35,0.35,0.15$ \\
\hline
\end{tabular}

\section{Results}

It can be noted that, for the case M1 (Table 2), the DWCS-1 sequence has the lowest energy requirement and $\mathrm{CO}_{2}$ emissions, but the DWCS-4 presents the minimum total annual cost and the highest thermodynamic efficiency. This result is in agreement with the fact that the optimum scheme must be selected in terms of the total annual cost, because the same energy requirements in complex distillation sequences can be 
Design and Control of New Intensified Distillation Systems for Quarernary Separations using Genetic Algorithms

translated into different costs because of their dependence on the temperatures of the integrated distillation sequence reboilers. The worst option in TAC value is DWCS-3. The analysis was conducted on the other cases of study and it can be established that, in general, DWCS-1 sequence has the lowest energy consumption and $\mathrm{CO}_{2}$ emissions, but the better option in TAC and $\eta$ values is DWCS-4. A preliminary heuristic rule can be suggested: the best option is presented when the component $\mathrm{A}$ is purified in a conventional sequence. The study is complemented by a dynamic analysis (closed-loop using PI controllers). We attempted a common ground for comparison by optimizing the controller parameters, proportional gains $\left(\mathrm{K}_{\mathrm{C}}\right)$ and reset times $(\tau \mathrm{i})$, for each conventional and integrated scheme following the integral of the absolute error (IAE) criterion.

Table 2. Energy consumption, total annual cost, thermodynamic efficiency and $\mathrm{CO}_{2}$ emissions for M1.

\begin{tabular}{lllll}
\hline Sequence & $\begin{array}{l}\text { Energy } \\
\text { consumption } \\
(\mathrm{BTU} / \mathrm{hr})\end{array}$ & $\begin{array}{l}\text { Total } \\
\text { Annual }\end{array}$ & $\begin{array}{l}\text { Thermodynamic } \\
\text { efficiency } \\
\text { Cost (USD) }\end{array}$ & $\begin{array}{l}\mathrm{CO}_{2} \\
\text { Emissions } \\
(\mathrm{kg} / \mathrm{hr})\end{array}$ \\
\hline DWCS-1 & $2,529,918.70$ & $531,287.1$ & 21.4 & 180.7 \\
\hline DWCS-2 & $3,436,258.40$ & $553,691.7$ & 19.6 & 245.4 \\
\hline DWCS-3 & $2,537,126.50$ & $680,975.9$ & 21.8 & 181.2 \\
\hline DWCS-4 & $2,644,464.70$ & $499,941.4$ & 22.1 & 193 \\
\hline DWCS-5 & $3,521,712.80$ & $579,412.7$ & 17.6 & 251.5 \\
\hline
\end{tabular}

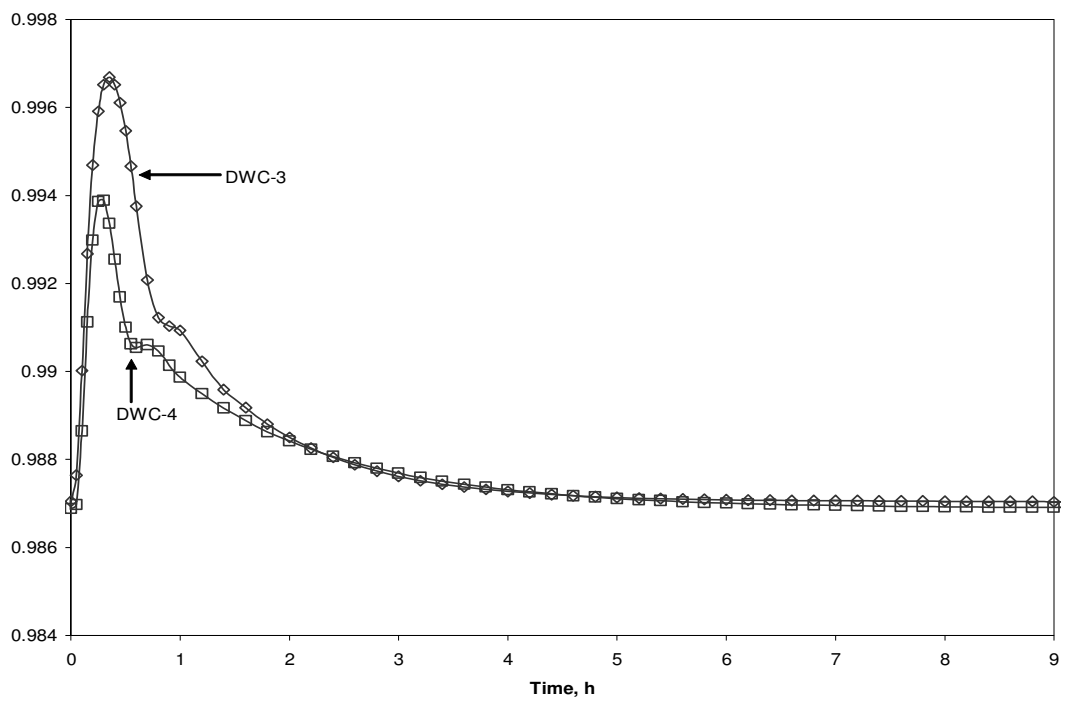

Figure 2. Dynamic responses for a feed disturbance in component A. 
The liquid compositions for the main product streams (A, B, C and D) are taken as the controlled variables. The control loops for the thermally coupled columns were chosen from an extension of the energy balance configuration for conventional distillation column. When a feed disturbance was implemented, intensified systems successfully rejected the disturbance to bring the product composition back to its design value. However, the responses of the DWCS-4 and DWCS-2 were remarkably better in comparison with DWCS-3 (Figure 2). This result is important: the best sequence in $\mathrm{TAC}$ value is the best in dynamic behavior.

\section{Conclusions}

A design methodology for intensified thermally coupled distillation sequences has been presented. The design and optimization methodology used have proven to be an important tool to resolve these kinds of problems, producing results close to the global optimum and with low mathematical effort. Because of the complex nature of the studied systems, this rigorous simulation method is absolutely necessary to ensure that the best solution is chosen. As it can be seen from the results, the best option is the DWCS-4 in TAC and $\eta$ values. The control properties show good dynamic closed-loop performance in the best option.

\section{Acknowledgements}

We acknowledge the financial support provided by Universidad de Guanajuato, CONACyT and CONCyTEG (Mexico).

\section{References}

[1] Stankiewicz, A., Moulijn, J. A., 2000, Process intensification: Transforming chemical engineering, CEP, 96, 22.

[2] Amminudin, K.A., Smith, R., Thong, D.Y.C., Towler, G.P., 2001, Design and Optimization of Fully Thermally Coupled Distillation Columns. Part 1: Preliminary Design and Optimization Methodology. Chem. Eng. Res. Des., 79: 701.

[3] Schultz, M.A., Stewart, D.G., Harris, J.M., Rosenblum, S.P., Shakur, M.S., O'Brien, D.E., 2002, Reduce Costs with Dividing Wall Columns. Chem Eng Prog, May, 64.

[4] Adrian, T., Schoenmakers, H., Boll, M., 2004, Model Predictive Control of Integrated Unit Operations: Control of a Divided Wall Column. Chem Eng Process, 43, 347.

[5] Gutiérrez-Antonio, C., Briones-Ramírez, A., 2009, Pareto front of ideal Petlyuk sequences using a multiobjective genetic algorithm with constraints, Computers and Chemical Engineering, in press. 